\title{
Polipropileno reforzado con fibra natural para fabricación de paneles internos de las puertas de un automóvil
}

\section{Polypropylene reinforced with natural fiber for the manufacture of interior panels of the doors of an automobile}

\author{
Manuel Fernando Gómez Berrezueta \\ Universidad Internacional del Ecuador, Ecuador \\ Paúl Wilfrido Méndez Torres \\ Universidad Politécnica Salesiana, Ecuador
}

Autor para correspondencia: fernanditogb@hotmail.com, magomezbe@uide.edu.ec, pwmt1979@gmail.com,pmendez@ups.edu.ec

Fecha de recepción: 10 de Agosto de 2017 - Fecha de aceptación: 30 de Septiembre de 2017

Resumen: Este artículo presenta las características técnicas, económicas y ambientales a considerar para fabricar una pieza de uso automotriz, que cumplan con las especificaciones. En este estudio, se discuten las propiedades y ventajas de los compuestos de fibra natural en paneles de puertas interiores. Las fibras naturales están reemplazando otros materiales en varias partes de automóviles debido a su peso ligero, bajo costo, bajo $\mathrm{CO}$, reciclabilidad. El objetivo de este proyecto es que los componentes cumplan los siguientes requisitos: simplicidad de construcción, facilidad de fabricación, colocación de materiales y bajo costo. El documento describe los efectos de varios parámetros materiales tales como tratamientos de la fibra, composición microestructural, técnicas de fabricación de compuestos, propiedades técnicas, cantidad de fibra a añadir establecida en\% de peso, tipo de polímero, etc.; a varios niveles de contenido de fibra. Las pruebas de Charpy permitieron establecer la influencia de la fibra sobre la resiliencia del material compuesto y microscopía electrónica de barrido (SEM) para analizar el tipo de fractura producida. Todos los ensayos están en Normas Internacionales de ASTM o Estándares Internacionales de ISO. Finalmente, los datos experimentales indican que los materiales procesados con fibras naturales (fibras de yute-kenaf) tienen un mejor rendimiento mecánico global. En esta aplicación pueden mejorar económicamente el rendimiento cuando se usan termoplásticos no reforzados convencionales. Los compuestos de fibra-polipropileno naturales tienen algunos inconvenientes inherentes: el procesado y la materia prima.

Palabras Clave: bajo costo; características físicas; compuesto; fibra natural; microestructura; paneles interiores; puertas de automóviles; polímero; polipropileno; propiedades mecánicas; reciclable; yute

Abstract: This article presents the characteristics technical, economic and environmental to consider to make a piece of automotive use considerations, namely that meet the specifications. In this study, the properties and advantages of natural fiber composites in inner door panels are discussed. Natural fibers are replacing other materials in various parts of automobiles due to their lightweight, low cost, low $\mathrm{CO} 2$, recyclability. The goal of this project is the components that meet 
the following requirements: construction simplicity, ease of manufacturing, placement of materials and low in cost. The paper describes the effects of several material parameters such as fiber treatments, microstructural composition, composite-manufacturing techniques, techniques properties, quantity of fiber to be added established in \% weight, polymer type, etc. A several levels of the fiber content. The important of the tests: tensil and bending experiments have been carrying out to evaluate the resistance and plasticity, respectively, Charpy tests allowed establishing the influence of the fiber on the resilience of the composite, scanning electron microscopy (SEM) to analyze the type of fracture produced. All tests are in ASTM International Standards or ISO International Standards. Finally experimental data indicate that the materials processed with natural fibers (Jute-Kenaf fibers) has a better global mechanical performance. In this application they can economically improve performance when conventional unreinforced thermoplastics are used. Natural fiber-polypropylene composites have some inherent drawbacks: processing and feedstock.

Key words: low cost; physical characteristics; composite; natural fiber; microstructure; interior anels; car doors; polymer; polypropylene; mechanical properties; recyclable, jute

\section{Introducción}

Los materiales plásticos, por lo general polímeros, son comúnmente usados en diferentes ramas de la ingeniería como: arquitectura, eléctrica, aeronáutica y automotriz. Siendo la industria automotriz, diseño y fabricación de partes del vehículo, la que más se ha desarrollado en los últimos años, debido a las exigencias de las restricciones sobre el medio ambiente. Siendo necesario el uso de materiales plásticos reforzados con fibras sintéticas y/o naturales. Las fibras naturales como: lino, yute, sisal, corteza de banano, entre otras; se ha usado en algunos componentes automotrices como: paneles interiores de puertas, espaldares, paneles de instrumentos, apoyacabezas, guardafangos, consolas y otros; por brindar algunas ventajas: más bajo costo, renovables y biodegradables. Estas fibras son procedentes de diferentes partes del mundo, principalmente donde existe clima cálido. El reemplazo de materiales como el aluminio, el acero, plásticos reforzados con fibras sintéticas (vidrio, carbono) permite la disminución de la masa y ahorro de combustible. Presentan una muy buena resistencia mecánica (Cristaldi, 2010). Esta tendencia empezó en Europa, luego continuó en EEUU y Asia.

En base a las necesidades y especificaciones técnicas de la aplicación ingenieril a fabricar, en este caso un panel interior de las puertas de un automóvil, se debe analizar las condiciones de funcionamiento de la pieza, de manera individual y en conjunto; tomando en cuenta aspectos técnicos de diseño y fabricación. Como todo elemento de uso automotriz debe pasar por rigurosos métodos de evaluación y pruebas, para cumplir con las especificaciones: de seguridad, rendimiento, medioambientales, económicos, etc. En lo referente a las especificaciones técnicas más significativas de la pieza a fabricar se consideran: resistir a esfuerzos y cargas durante el impacto de tipo lateral, una óptima resistencia térmica, tener un buen acabado superficial, cumplir condiciones funcionales y dimensionales (específicos de acuerdo al tipo y clase de vehículo), resistencia a los golpes y fatiga, reacción favorable a factores medioambientales como humedad, rayos solares, materiales extraños (polvo, aceites, etc.). Aspectos como las prestaciones mecánicas, la resistencia térmica, o la emisión de componentes volátiles responsables de la aparición de olores en el interior de los vehículos deben mejorarse para los materiales usados en partes internas del vehículo. 
Dada la importancia de alcanzar estándares de seguridad en los vehículos y su relación costo/beneficio existen algunas regulaciones que limitan uso de ciertos materiales, obligando así, a la búsqueda de nuevas alternativas en el uso de materiales para la fabricación de ciertas piezas del automóvil.

Las regulaciones de Seguridad de los vehículos, establecen pruebas y estándares que deben cumplir los vehículos, es así en lo referido a las puertas y su componentes establecen en EEUU, según FMVSS (Federal Motor Vehicle Safety Standards) Parte 571- Resistencia al impacto, estipula en los estándares 201 y 214, lo relacionado con Protección de los ocupantes en impacto interno y Protección en impacto lateral; respectivamente (Figura 1 y Figura 2). También en la Parte 572-Sección F- Pruebas Dinámicas: de impacto lateral usando dummies (SIDs)FMVSS 214 (Side Door Streength), para cumplir con las regulaciones del Departamento del Transporte de Estados Unidos (U.S. DOT). En Europa existen pruebas similares regidas por EuroNCAP.

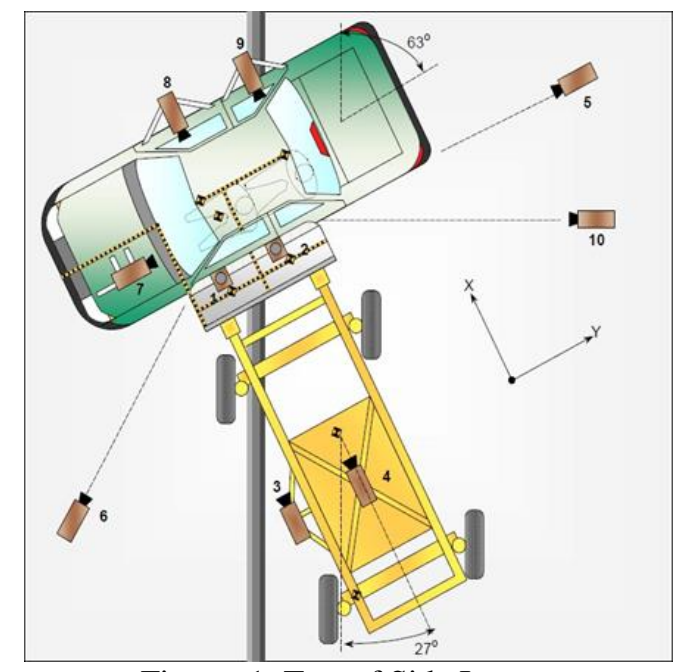

Figura 1. Test of Side Impact

Recuperado de http://www.nhtsa.gov/cars/rules/import/FMVSS/

En Europa según 2000/53/EC, normativa relacionada con la vida útil y reutilización de los vehículos, determinó que para el 2006, el 80\% del vehículo debe ser reciclado y reusado, aumentando al $85 \%$ en el 2015. En Japón era de $88 \%$ en el 2005 y debe alcanzar el $95 \%$ en el 2015 (Holbery, 2006). 


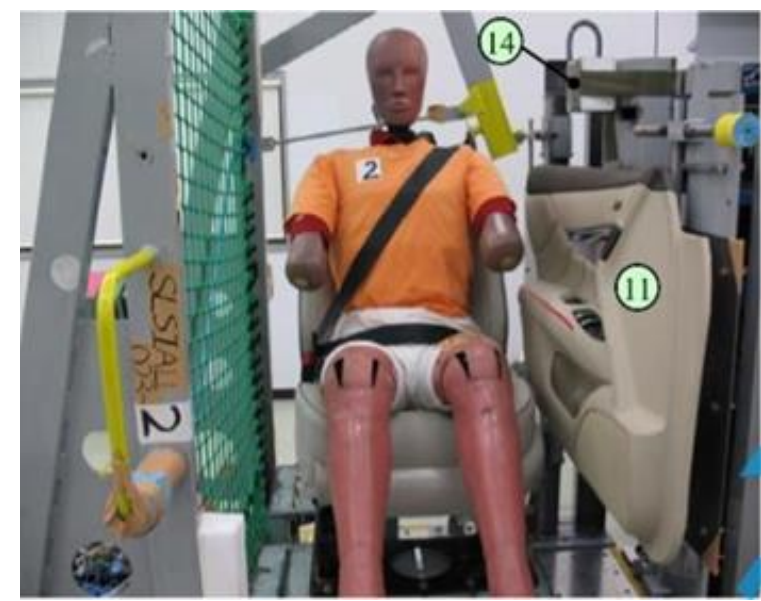

Figura 2. Schematic of test device. Recuperado de https://www.euroncap.com/es

Determinado las condiciones de servicio de la pieza a fabricar, su comportamiento estático y dinámico, se debe satisfacer por parte del material compuesto (polímero/fibra natural) elegido ciertas características: mecánicas, físicas, químicas, ambientales y de fabricación (Saheb, 1999). Se analiza desde el punto de vista técnico las mejores opciones que existen para la elaboración de dicho material, considerando la disponibilidad de varios polímeros y así mismo de varias fibras naturales.

Considerando a las fibras como el material innovador del compuesto, se determina sus características principales, considerando aspectos de obtención, tratamiento, procedimiento, fabricación, pruebas y análisis. Uno de los aspectos que se considera es la proporción con el polímero y la forma de fabricación, de manera de obtener un material adecuado para su procesado mediante las tecnologías de moldeo por inyección y termoconformado, procesos que mayoritariamente son usados en el sector automotriz, y que cumpla con los requerimientos para su empleo en el interior de los vehículos (Mueller D. H., 2004).

Una vez elegido la fibra y el polímero, se analiza el comportamiento del mismo, su estructura, formas, características, se realiza un estudio de las alternativas de compuestos (polímero/fibra natural), que puedan determinarse las pruebas, procedimientos y resultados. Las pruebas se realizan de acuerdo a estándares internaciones de instituciones como ASTM, ISO, SAE principalmente. (Nur, 2010)

Los problemas se presentan en la unión o cohesión entre la fibra y el polímero, y el posible surgimiento de posibles mecanismos de falla.

Las ventajas de usar fibras naturales respecto a otros materiales, incluso otras fibras es muy significativo (Figura 3). 


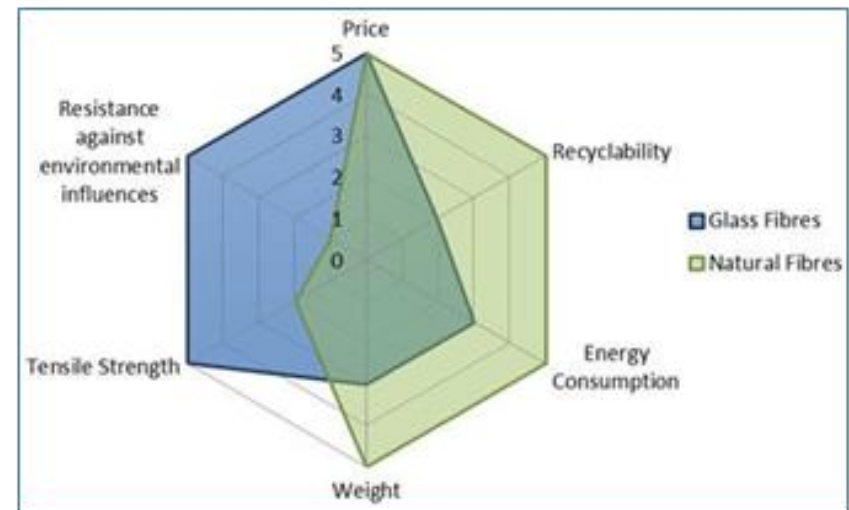

Figura 3. Relative merits of natural fibres composites. Recuperado de (Cristaldi, 2010)

Es por ello que las organizaciones de normalización, como la Sociedad Americana para Pruebas y Materiales (ASTM) y la Organización Internacional de Normalización (ISO) han desarrollado estándares para medir el contenido de base biológica y la realización de evaluaciones del ciclo de vida.

\section{Descripción de la Aplicación}

Los paneles interiores de los vehículos Fig. 3, al igual que el resto de componentes de un automóvil deben cumplir ciertas características y especificaciones técnicas, para lo cual existe basta normativa al respecto (Holbery, 2006). Como ya se ha mencionado, la aplicación dónde se implementará el material compuesto por: un polímero y una fibra natural; es un componente de la puerta del automóvil, el panel interior; que cumple con los siguientes requisitos:

- Simplicidad de construcción.

- Facilidad de fabricación y colocación de materiales.

- Alta rigidez y seguridad.

La pieza a fabricar es un panel interior de las puertas del automóvil (Figura. 4).

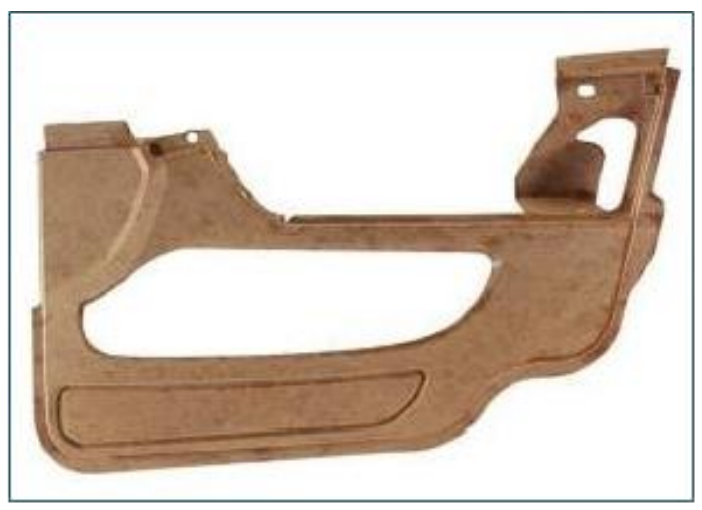

Figura 4. Inner Door Panel Recuperado de (Holbery, 2006)

Este elemento del vehículo debe cumplir las especificaciones técnicas de diseño y construcción, principalmente con las normas de seguridad, referidas al impacto lateral y condiciones de acoplamiento y dimensionado. 
Son algunas las características que deben cumplir las partes interiores del vehículo y, específicamente; los paneles internos de las puertas, mismas que se mencionan a continuación:

- Resistir a los impactos.

- Tener peso reducido y ser biodegradable.

- No producir ruido ni vibraciones.

- Estar dimensionalmente óptima, acorde al tipo de vehículo (Dimensiones del habitáculo).

- Durabilidad y fácil recambio.

- Resistir los efectos medioambientales, principalmente la humedad y calor; producidos en el interior del vehículo.

- Acabado aceptable.

- Bajo costo.

En la Tabla 1 se observa los pesos promedios de las partes internas de los automóviles, usando compuestos con refuerzos de fibras naturales.

Tabla 1

Weight of Automotive's

\begin{tabular}{ll}
\hline Component & Weight $(\mathbf{K g})$ \\
\hline Front door lines & $1.2-1.8$ \\
Rear door lines & $0.8-1.5$ \\
Boot liners & $1.5-2.5$ \\
Parcel shelves & $<2$ \\
Seat backs & $1.6-2$ \\
& \\
Sunroof shields & $<0.4$ \\
Headrest & 2.5 \\
\hline
\end{tabular}

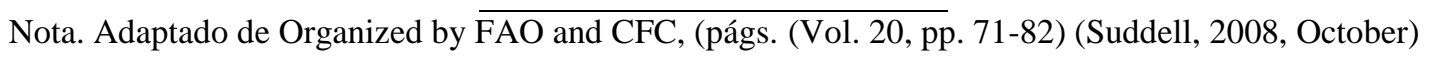

Actualmente, algunas marcas de vehículos ya fabrican un determinado número de piezas usando materiales compuestos con fibras naturales (Fig. 4). Con este fin se han utilizado estos materiales en el interior de una gran cantidad de vehículos. Como ejemplo, en 1996 MercedesBenz usó una matriz epoxy con de fibras de yute para los paneles de las puertas en vehículos clase E y en el 2000 Audi usó poliuretano reforzado con fibras de lino y sisal para recubrimientos de puertas.

Tomando en consideración las ventajas ofrecidas por las fibras naturales, su uso es más frecuente en las partes internas del vehículo, ya que disminuye el peso del vehículo y son más económicas. En las industrias manufactureras de automóviles el objetivo final es reducir hasta un $30 \%$ en peso y un $20 \%$ en costo (Ahmad, 2015). 


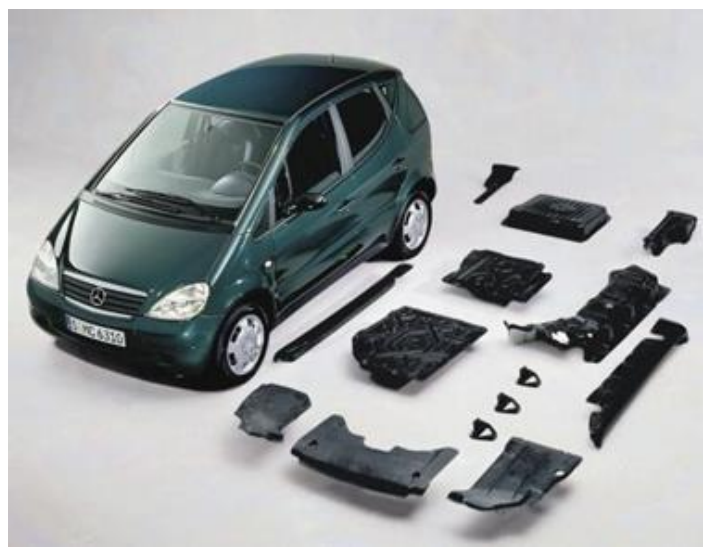

Figura 5. Automotive components made of natural fiber-reinforced composites Recuperado de http://www.sciencedirect.com/science/article/pii/S1369702103004292

\section{Requerimientos del material}

Los materiales de uso ingenieril, en el campo automotriz, como todos exigen algunas características que deben cumplir.

Las propiedades de algunas de las fibras naturales pueden observarse en la Tabla II.

Tabla 2

Chemical composition of natural fibers

\begin{tabular}{ll} 
Fiber & Cellulose (Wt \%) \\
\hline Banana & $63-64$ \\
Cotton & $85-90$ \\
Flax & 71 \\
Hemp & 68 \\
Henequen & 60 \\
Jute & $61-71$ \\
Sisal & 65 \\
\hline \multicolumn{2}{l}{ Recuperado de (John, 2008) }
\end{tabular}

Las fibras naturales contienen $60-80 \%$ de celulosa, 5-20\% de lignina y hasta un $20 \%$ de humedad (Saheb, 1999).

Tabla 3

Chemical composition

\begin{tabular}{|c|c|c|c|c|c|}
\hline Fibers & Diameter $(u \mathbf{m})$ & Density $\left(\mathrm{kg} / \mathrm{m}^{3}\right)$ & Cell & l/d ratio & Microfibrilla r Angel (ø) \\
\hline Banana & $80-250$ & 1350 & 150 & & $10+-1$ \\
\hline Sisal & $50-220$ & 1450 & 100 & & $10-22$ \\
\hline Pineapple leaf & $20-80$ & 1440 & 450 & & $8-14$ \\
\hline Palmira & $70-1300$ & 1090 & 43 & & $29-32$ \\
\hline
\end{tabular}

Adaptado de (ElayaPerumal, 2008) 
Las propiedades físicas de las fibras naturales se determinan principalmente por la composición química y física, tal como la estructura de fibras, contenido de celulosa, el ángulo de las fibrillas, sección transversal, y por el grado de polimerización (Tabla III). Sólo unos pocos valores característicos, pero especialmente las propiedades mecánicas específicas, pueden alcanzar valores comparables de fibras de refuerzo tradicionales.

Debido a los esfuerzos que va a estar sometido es necesario cumplir con ciertas propiedades físicas y mecánicas del material.

La resistencia a la tracción y el módulo de Young de las fibras aumenta con el porcentaje de celulosa. El ángulo de las microfibrillas determina la rigidez de las fibras (George, 2001) (Ahmad, 2015). La Tabla IV, presenta las propiedades físicas y mecánicas importantes de los diferentes tipos de fibras.Otro factor a considerar es el costo del material y el proceso de cultivo, tratamiento y producción.

Las fibras naturales son fibras renovables cultivadas en campos y pueden ser utilizadas como refuerzo en la manufactura de un material compuesto de la misma manera que una fibra sintética. Algunos ejemplos de las fibras más utilizadas son: abacá, cáñamo, lino, yute y ramina.

Las fibras de yute son las más cultivadas en todo el mundo con excelentes prestaciones mecánicas. Así mismo, el lino es una de las fibras relativamente más fuerte y rígida y más demandadas en Europa.

Las fibras naturales son más económicas y livianas para reducir el peso del automóvil (Mohanty, 2002).

Tabla 4

Physical and mechanical properties of selected natural fibers

\begin{tabular}{lllll}
\hline $\begin{array}{l}\text { Fiber } \\
\text { name }\end{array}$ & Density (g/cm3) & $\begin{array}{l}\text { Tensile } \\
\text { Strength }(\mathbf{M P a})\end{array}$ & $\begin{array}{l}\text { Tensile } \\
\text { Modulus }(\mathbf{G P a})\end{array}$ & $\begin{array}{l}\text { Elongation at break } \\
(\%)\end{array}$ \\
\hline Banana & 1.35 & 600 & 17.85 & 3.36 \\
Sisal & 1.45 & $530-640$ & $9.4-22$ & $3-7$ \\
Jute & 1.46 & $393-800$ & $10-30$ & $1.5-1.8$ \\
Flax & 1.5 & $800-1500$ & $27.6-80$ & $1.2-3.2$ \\
\hline
\end{tabular}

Adaptado de (Ahmad, 2015)

\section{Ventajas de las fibras naturales}

La versatilidad de las fibras naturales se basa en las siguientes propiedades:

- Fibras vegetales son una materia prima renovable y su disponibilidad es más o menos ilimitada.

- Tiene muy buenas propiedades mecánicas, especialmente la fuerza de tracción. En relación a su peso, las mejores fibras, pueden alcanzar fuerza similar a la de Kevlar. 
- Posee buen desempeño como aislante térmico, acústico y propiedades de aislamiento eléctrico.

- Combustibilidad: productos pueden ser eliminados a través de la quema al final de su vida de servicio útil y se puede generar energía al mismo tiempo.

- Biodegradabilidad: como resultado de su tendencia a absorber agua, fibras naturales se biodegradan en determinadas circunstancias (hongos y / o bacterias).

- Su naturaleza abrasiva es mucho menor en comparación con la de fibra de vidrio, lo que conlleva a, un reciclado de material técnico o procesamiento de materiales compuestos en general.

- Reactividad: los grupos hidroxilo que están en los constituyentes de la pared celular no sólo proporcionan sitios para la absorción de agua, sino también están disponibles para modificación química (por ejemplo, introducir estabilidad dimensional, durabilidad o mejoradas propiedades de absorción de aceite / metal pesado). (ElayaPerumal, 2008)

Desventajas de las fibras naturales

A continuación se enlistan algunos de los inconvenientes de las fibras naturales:

- Propiedades de resistencia más bajos, en particular su resistencia al impacto.

- La variabilidad en la calidad, en función de las influencias impredecibles como el clima.

- La absorción de humedad, lo que provoca la inflamación de las fibras.

- La máxima temperatura de procesamiento es restringida.

- Baja durabilidad, se puede mejorar efectuándole tratamientos.

- Poca resistencia al fuego.

- A consecuencia de la higroscopicidad de las fibras, productos y material a base de fibras vegetales no son dimensionalmente estable bajo condiciones de humedad cambiante.

Al realizar un análisis de las diferentes propiedades de los materiales se puede obtener una comparación de las fibras naturales, respecto a otros materiales en este caso las fibras sintéticas (fibra de vidrio) que también son usadas en la fabricación de los componentes analizados.

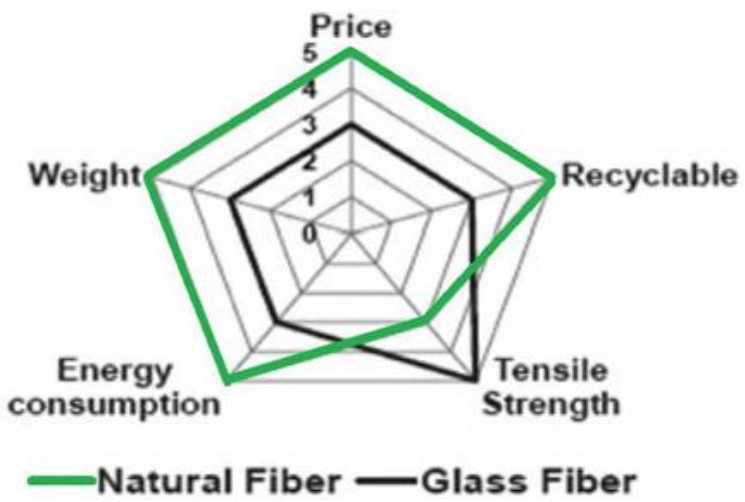

Figura 6. Comparison between natural fiber and glass fiber Adaptado de (Faruk, Biocomposites reinforced with natural fibers: 2000-2010. Progress in Polymer Science, 2012. 37(11)) 


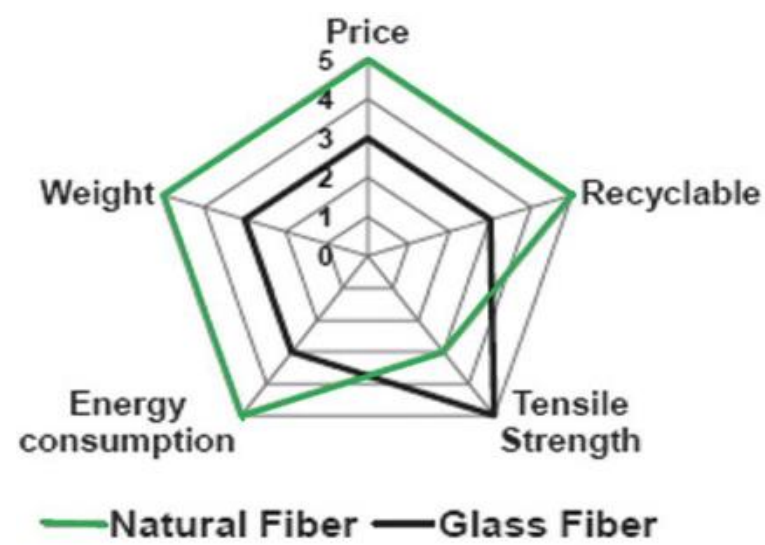

Figura 7. Comparison between natural fiber and glass fiber Adaptado de (Faruk, Biocomposites reinforced with natural fibers: 2000-2010. Progress in Polymer Science, 2012. 37(11))

En las Figura 6 y Figura 7, se compara las fibras naturales con respecto a la fibra de vidrio. Se puede observar que los objetivos principales del componente a fabricar son el precio, reutilizables y un esfuerzo medio. En la Figura 8 se ve también graficada lo referente a la fibra de carbono, material muy utilizado; sobre todo en vehículos de alta gama.

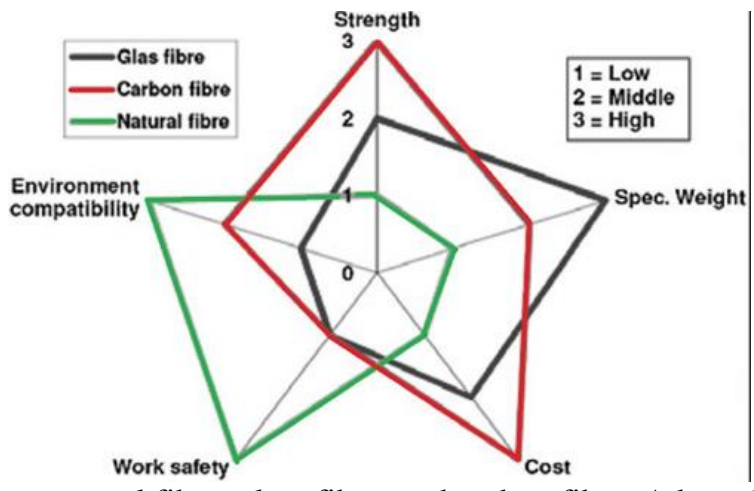

Figura 8. Comparison between natural fiber, glass fiber, and carbon fiber. Adaptado de (Faruk, Biocomposites reinforced with natural fibers: 2000-2010. Progress in Polymer Science, 2012. 37(11))

Realizando un análisis en general se puede ver las ventajas respecto a los factores técnicos, económicos, ambientales y sociales (Figura 9).

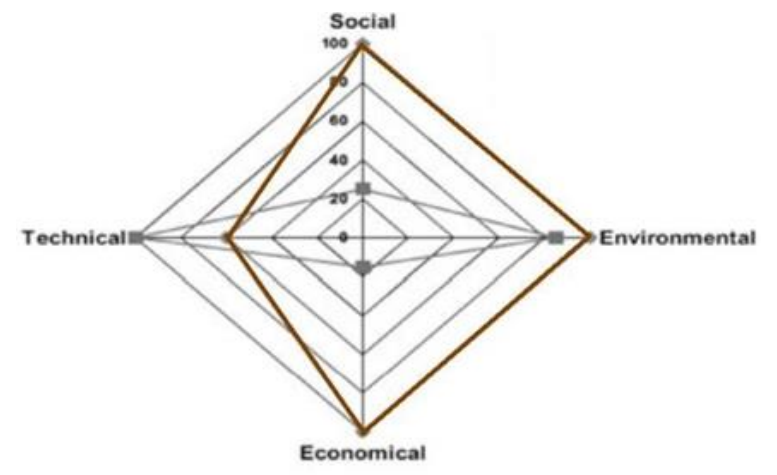

- Jute Composites - - -Glass Composites

Figura 9. Comparison between jute and glass composites based bonnet's aspects. Adaptado de (Faruk, Biocomposites reinforced with natural fibers: 2000-2010. Progress in Polymer Science, 2012. 37(11)) 
Como ejemplo de consumo de energía para producir un compuesto de un fibra de lino (flax) la energía consumida es de aproximadamente $9.55 \mathrm{MJ} / \mathrm{kg}$, incluido el cultivo, curación y separación; en comparación con los $54.7 \mathrm{MJ} / \mathrm{kg}$, que consume una fibra de vidrio. Datos de consumo de energía, de diferentes fibras podemos observar en la Figura 10 y costos por el peso en la Figura 11.

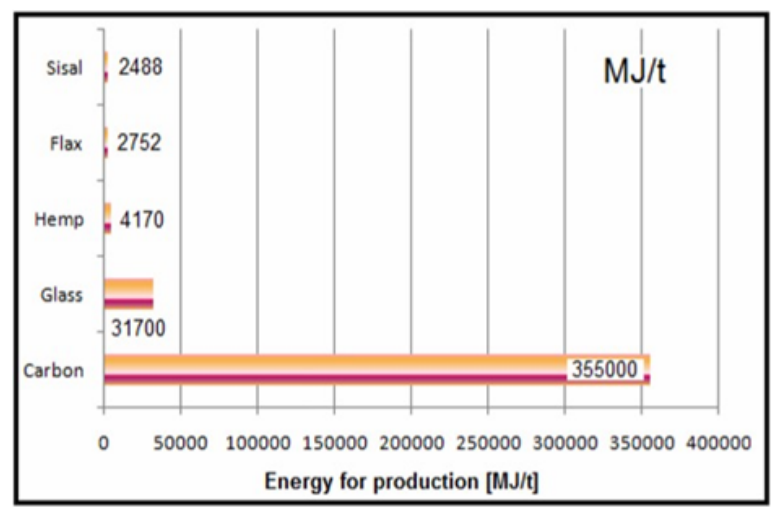

Figura 10. Energy for production of fibers Recuperado de (Faruk, Progress report on natural fiber reinforced composites , 2014)

Otro factor a considerar es el costo del material, ya que la mayoría de fibras si cumple con algunas de las propiedades físicas y químicas solicitadas.

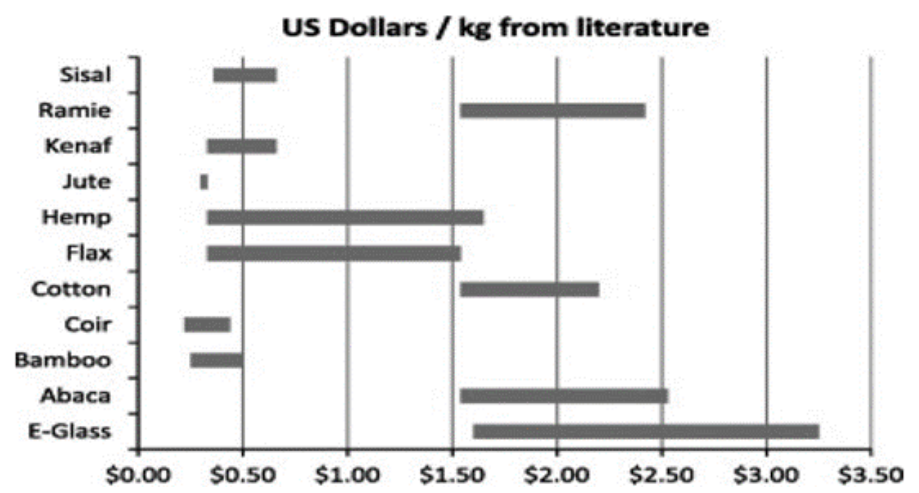

Figura. 11. Cost per weight comparison Recuperado de (Faruk, Progress report on natural fiber reinforced composites , 2014)

A continuación se analiza el polímero que mejores prestaciones proporciona para la elaboración de la pieza a fabricar, se realiza una comparación entre los termoplásticos y los termofijos, que son los polímeros que más comúnmente se utilizan en compuestos con fibras naturales. En la Tabla 5 puede observarse las ventajas y desventajas, entre ellos.

Tabla 5

Thermoplastics vs Thermosets

\begin{tabular}{ll}
\hline Thermoplastics & Thermosets \\
\hline Pros & Pros \\
High Impact Strength & Easy to process and laminate
\end{tabular}


Attractive Surface Finish

Recyclable / Scrap is Reusable

No Emissions

Can bond to other thermoplastics

Can be molded or shaped by reheating

Cons

Typically will soften with heat

More difficult to prototype
Does not necessarily need pressure or heat to form

Typically inexpensive

Typically stronger than thermoplastics

Better suited to higher temperatures than thermoplastics

\section{Cons}

Often release emissions known as volatile organic compounds (VOCs)

Non-recyclable and cannot be reclaimed easily

Short workable pot life, with some exceptions

Analizando las características de los diferentes polímeros, se optó por el Polipropileno (PP), por ofrecer las siguientes características idóneas para la aplicación automotriz a usarse:

- Excelentes propiedades físicas, químicas, mecánicas, térmicas y eléctricas

- Bajo en peso.

- Excelente resistencia a las manchas, disolventes orgánicos, y desengrasado.

- Resistencia al ataque electrolítico.

- Baja tasa de absorción de humedad.

Resaltándose el hecho de su economía, su baja densidad y resistencia a los golpes e impactos, factores objetivos de la aplicación donde se utilizará el compuesto.

El polipropileno a nivel molecular se compone de una cadena principal de átomos de carbono enlazados entre sí a las cuales se les añaden grupos metilo ambos lados (Hill, 2012).

Las matrices poliméricas más usadas como compuestos de fibras son las siguientes (Faruk, Biocomposites reinforced with natural fibers , 2012): base petroquímica como los termoplásticos (polipropileno (PP), polietileno (PE), poli estireno (PS) y PVC Cloruro de polivinilo) y termo fijos (poliéster, resina epóxica, fenol formaldehido, éster de vinilo). Tabla 6. 
Tabla 6

Properties of Polymers

\begin{tabular}{|c|c|c|c|c|}
\hline Property & PP & LDPE & HDPE & PS \\
\hline Density $\left(\mathrm{g} / \mathrm{cm}^{3}\right)$ & $0.899-0.920$ & $0.910-0.925$ & $0.94-0.96$ & $1.04-1.06$ \\
\hline $\begin{array}{l}\text { Water Absorption } \\
-24 \text { hours }(\%)\end{array}$ & $0.01-0.02$ & $<0.015$ & $0.01-0.2$ & $0.03-0.10$ \\
\hline $\mathrm{T}_{\mathrm{g}}\left({ }^{\circ} \mathrm{C}\right)$ & -10 to -23 & -125 & -133 to -100 & - \\
\hline $\mathrm{T}_{\mathrm{m}}^{\circ}\left({ }^{\circ} \mathrm{C}\right)$ & $160-176$ & $105-116$ & $120-140$ & $110-135$ \\
\hline $\begin{array}{l}\text { Heat Deflection } \\
\text { Temp }\left({ }^{\circ} \mathrm{C}\right)\end{array}$ & $50-63$ & $32-50$ & $43-60$ & Max. 220 \\
\hline $\begin{array}{l}\text { Coefficient of } \\
\text { Thermal Expansion } \\
\left(\mathrm{mm} / \mathrm{mm} /{ }^{\circ} \mathrm{C} \times 10^{5}\right)\end{array}$ & $6.8-13.5$ & 10 & $12-13$ & $6-8$ \\
\hline $\begin{array}{l}\text { Tensile Strength } \\
\text { (MPa) }\end{array}$ & $26-41.4$ & $40-78$ & $14.5-38$ & $25-69$ \\
\hline $\begin{array}{l}\text { Elastic Modulus } \\
\text { (GPa) }\end{array}$ & $0.95-1.77$ & $0.055-0.38$ & $0.4-1.5$ & $4-5$ \\
\hline Elongation (\%) & $15-700$ & $90-800$ & $2.0-130$ & $1-2.5$ \\
\hline $\begin{array}{l}\text { Izod Impact } \\
\text { Strength }(\mathrm{J} / \mathrm{m})\end{array}$ & $21.4-267$ & $>854$ & $26.7-1,068$ & 1.1 \\
\hline
\end{tabular}

Recuperado de (Holbery, 2006)

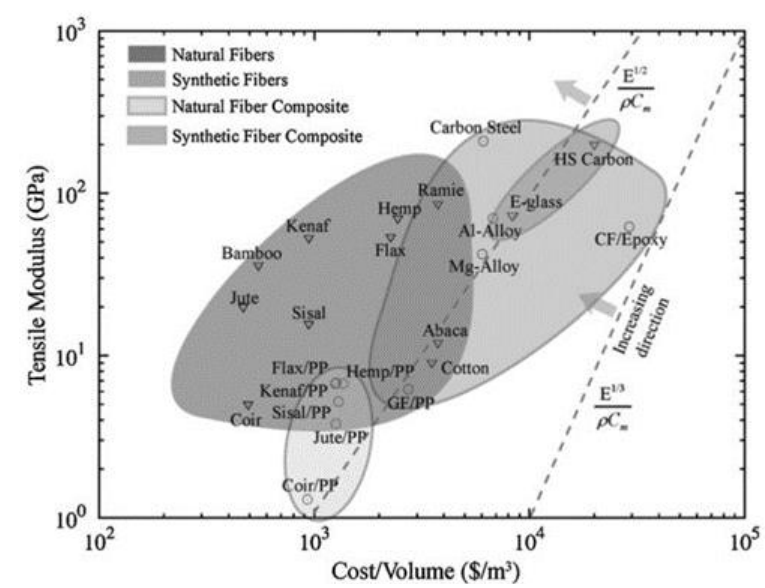

Figura 12. Tensile strength versus cost per volumen Recuperado de (Ashby, 1989)

En la Figura 12 se evalúa el módulo de tensión respecto al costo por unidad de volumen, y en la Figura 13 se puede apreciar la resistencia a la tensión respecto a la densidad del compuesto.

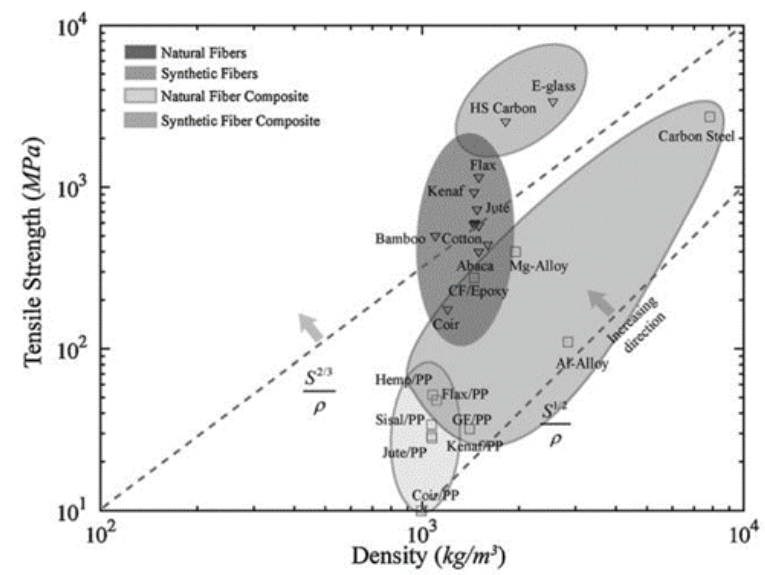

Figura 13. Tensile strength versus density diagram Recuperado de (Ashby, 1989) 


\section{Descripción del Material Propuesto}

Un material compuesto (composite) está formado principalmente por una combinación o mezcla de dos constituyentes que difieren en forma y composición química, y además por lo general son insolubles entre sí (Tabla 7). Estos materiales comprenden una fase continua llamada matriz y una discontinua llamada refuerzo (Satyanarayana, 2007). La matriz es la que contribuye a distribuir y estabilizar los esfuerzos y de proteger. Por otro lado la fase de refuerzo aporta las propiedades mecánicas al material.

Tabla 7

Properties

\begin{tabular}{ll}
\multicolumn{2}{c}{ Properties } \\
\hline Thermoplastic & Natural fibers \\
\hline Polypropylene & Curaua, flax, green coconut husks, \\
(PP) & hemp, jute, palm, sisal, wheat straw \\
Polyethylene & Banana, green coconut husks, rice husks, \\
(PE) & sisal, sugarcane bagasse \\
High & Banana, wood \\
polyethylene & density \\
(HDPE) & sisal, curaua \\
High impact polystyrene & green coconut husks \\
(HIPS) & sisal, sugarcane bagasse \\
\hline
\end{tabular}

Los materiales compuestos pueden clasificarse según el tipo de matriz en metálicos, cerámicos y poliméricos. La matriz usada en el proceso de fabricación de piezas de este tipo PMC.

Los refuerzos propuestos son fibras naturales, principalmente de cultivos agrícolas, para el presente artículo se consideró el cáñamo (Kenaf) y para ciertas pruebas el yute.

Para elaborar los paneles interiores de puertas del automóvil, se utilizó un polímero termoplástico: Polipropileno (PP).

\section{Fabricación del compuesto}

Hay que dirigir la fabricación de compuestos con fibras naturales a obtener propiedades superiores, que incluye la modificación química eficaz, eficiente y económica de la fibra, la modificación de la matriz de funcionalización y la combinación de técnicas de procesamiento. Por el lado la fibra se debe considerar el tipo, la composición química, las condiciones ambientales de crecimiento, los ángulos de las microfibras, los defectos, la estructura, las propiedades físicas y mecánicas y la interacción de la fibra con el polímero, entre otros.

\section{Técnicas de procesamiento}


Generalmente estos materiales son fabricados con técnicas tradicionales Figura 14, tales como: moldeo por compresión y/o moldeo por transferencia de resina RTM.

Las fibras se fraccionan y desbastan de la superficie de la fibra para mejorar su uniformidad y mejorar su potencial como refuerzo.

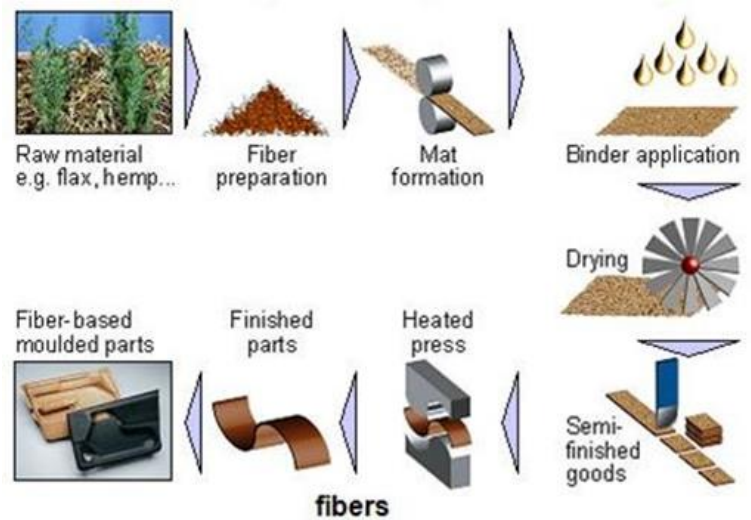

Figura 14. Fiber-based moulded parts Recuperado de http://www.globalhemp.com/2011/02/automotivecomposites.html

Dentro de los principales factores que influyen en el procesamiento tenemos:

Humedad relativa: (diseñado un nuevo tornillo extrusor -mayor L/D proporción, lo que permite una mejor desgasificación y en consecuencia menor contenido de humedad)

Tipo de fibra y contenido: longitud de las fibras, composición. Algunas formas de ubicarlas, unidireccionalmente, seguida de una forma tejida, en pequeñas partículas y por último de forma transversal (Lee, 2009).

\section{Propiedades y características}

El material propuesto debe cumplir ciertas propiedades, las cuales se evalúan en función de su uso interior, las especificaciones serán evaluadas de acuerdo a las normas de ensayo para comprobar las siguientes propiedades:

- Físicas: densidad, contenido de humedad, contenido de vacío, fracción de volumen, etc.

- Mecánicas: resistencia al impacto, tracción, etc.

- Durabilidad: envejecimiento artificial, hinchazón y absorción, etc.

- Térmicas: dilatación lineal, conductividad térmica, acción del calor.

- Fuego: límite de oxígeno, reacción al fuego.

- Otras propiedades

Tabla 8: Applications of Natural Fibers Composites-NFC in automobile 


\begin{tabular}{|c|c|c|}
\hline Manufacturer & Model & Application of NFC \\
\hline Audi & $\begin{array}{c}\text { A2,A3, A4, A4, } \\
\text { Avant, A6, A6 } \\
\text { Avant, A8, Roadster, } \\
\text { Coupe }\end{array}$ & $\begin{array}{l}\text { Seat backs, side and back door panel, boot lining. } \\
\text { hat rack, and spare tire lining }\end{array}$ \\
\hline BMW & $\begin{array}{l}3,5 \text {, and } 7 \text { series } \\
\text { and others }\end{array}$ & $\begin{array}{l}\text { Door panels, headliner panel, boot lining, seat backs, } \\
\text { noise insulation panels molded foot, and well linings }\end{array}$ \\
\hline Citroen & CS & Interior door paneling \\
\hline Daimler/Chrysler & $\begin{array}{l}\text { A, C, E, and S-Class, } \\
\text { EvoBus (exterior) }\end{array}$ & $\begin{array}{l}\text { Door panels, windshield, dashboard, business table, } \\
\text { and pillar cover panel }\end{array}$ \\
\hline Ford & Mondeo $\mathrm{CD} 162$, Focus & Door panels, B-pillar, and boot liner \\
\hline Lotus & Eco Elise & Body panels, spoiler, seats, and interior carpets \\
\hline Mercedes-Benz & Trucks & $\begin{array}{l}\text { Internal engine cover, engine insulation, sun visor, } \\
\text { interior insulation, bumper, wheel box, and roof cover }\end{array}$ \\
\hline Opel GM & Astra, Vectra, Zafira & $\begin{array}{l}\text { Headliner panel, door panels, pillar cover panel, } \\
\text { and instrument panel }\end{array}$ \\
\hline Peugeot & New model 406 & Seat backs and parcel shelf \\
\hline Renault & Clio, Twingo & Rear parcel shelf \\
\hline Rover & Rover 2000 and others & Insulation and rear storage shelf/panel \\
\hline Saab & - & Door panels and seat backs \\
\hline SEAT & - & Body panels, Spoiler, Seats, Interior carp, etc. \\
\hline TOYOTA & $\begin{array}{l}\text { Brevis, Harrier, } \\
\text { Celsior, RAUM }\end{array}$ & Door panels, seat backs, and spare tire cover \\
\hline Volkswagen & $\begin{array}{l}\text { Golf, Passat, Variant, } \\
\text { Bora, Fox, Polo }\end{array}$ & $\begin{array}{l}\text { Door panel, seat back, boot lid finish panel, } \\
\text { and boot liner }\end{array}$ \\
\hline Volvo & C70, V70 & Seat padding, natural foams, and cargo floor tray \\
\hline
\end{tabular}

Recuperado de (Faruk, Progress report on natural fiber reinforced composites , 2014)

Fracción de volumen de la fibra, contenido de Vacío y morfología de la superficie.

El contenido de vacíos (Figura 16) de las fibras naturales / PP compuestos se determinó utilizando la siguiente fórmula (ASTM D2734).

$$
\mathrm{V}=100(\mathrm{Td}-\mathrm{Md}) / \mathrm{Td}
$$

$\mathrm{V}$, Td, y Md fracción de volumen (vol. \%), densidad teórica $(\mathrm{g} / \mathrm{cm} 3)$ y densidad medida $(\mathrm{g} / \mathrm{cm} 3)$, respectivamente.

La superficie de fractura del espécimen fue probada usando un microscopio electrónico de barrido de superfice-SEM (Lee, 2009)

La Figura 15 muestra las fibras naturales que fueron recolectadas después de disolver la matriz de PP. 


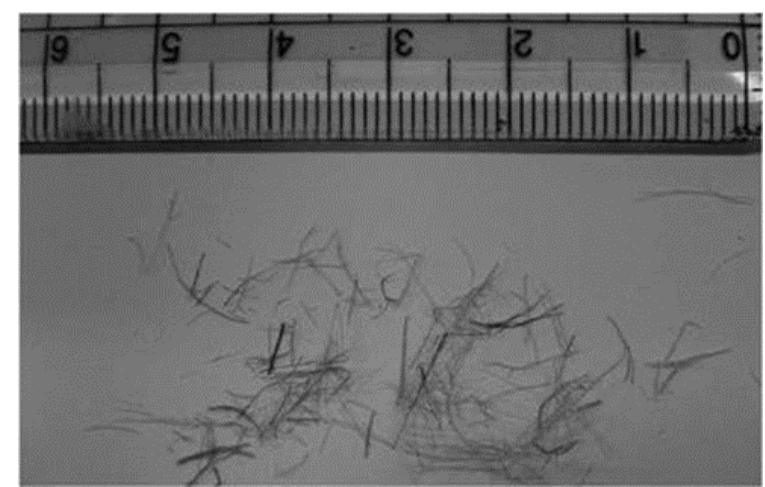

Figura 15. Natural fibers collected after dissolving PP matrix in composites. Recuperado de (Ahmad, 2015)

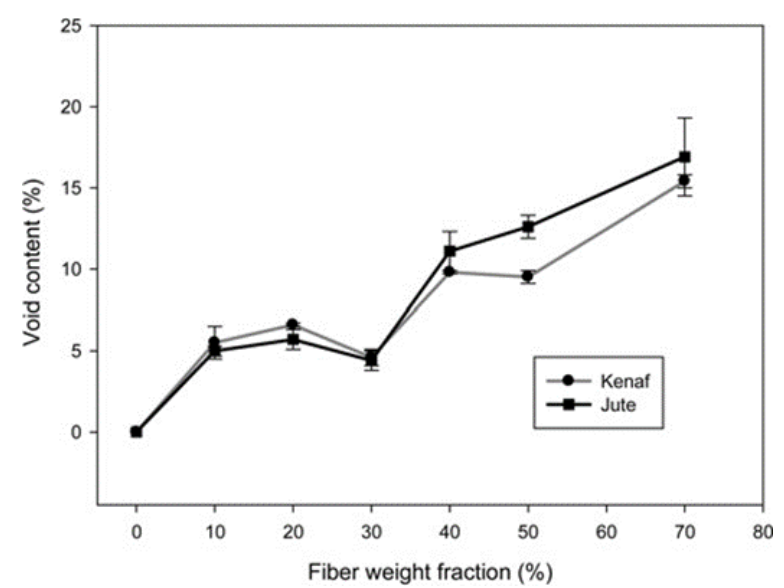

Figura 16. Void contents of natural fiber reinforced PP composites according to nominal fiber fraction by weight. Recuperado de (Lee, 2009)

\section{Composición química}

Las fibras naturales lignocelulósicas (Fig. 16) están compuestas principalmente de celulosa, hemicelulosa, lignina y pectina con una pequeña cantidad de extractivos (ver Figura17)

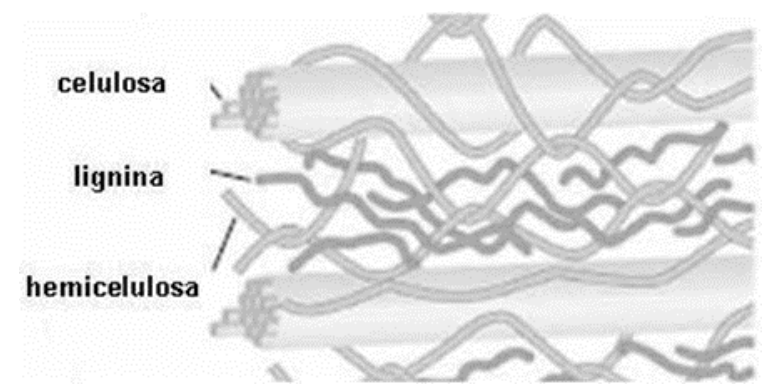

Figura 17. Composition of natural fiber Adapatado de (Schlöesser, 2004)

Según las características químicas de la fibra se obtienen mejoras en las propiedades a evaluar (Figura 18). 


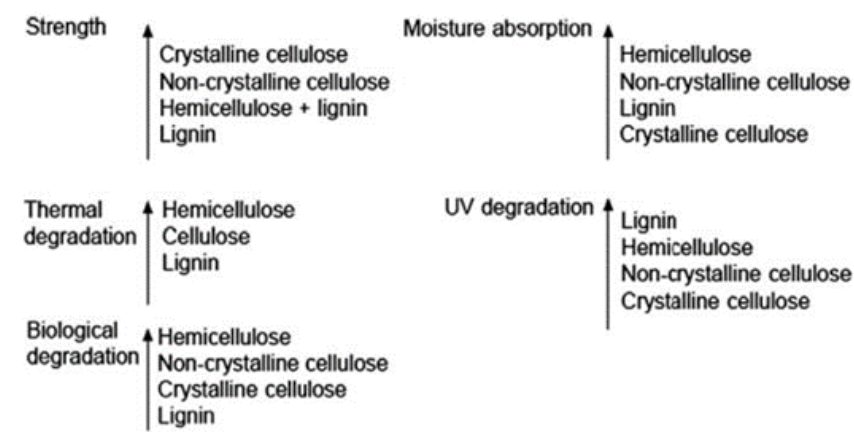

Figura 18. Properties of cellulose fibers and their dependence on chemical constituents. Adaptado de (Schlöesser, 2004)

\section{Estructura física}

Las fibras son básicamente una rígida celulosa cristalina, reforzada con microfibrillas amorfas de lignina y/o matriz de hemicelulosa, donde; la hemicelulosa actúa como una matriz de cementación entre las microfibrillas de celulosa, e incrementa la rigidez del compuesto hemicelulosa/celulosa (Figura 19). Las microfibrillas poseen un diámetro entre 10-30 nm y tienen entre 30-100 moléculas de celulosa.

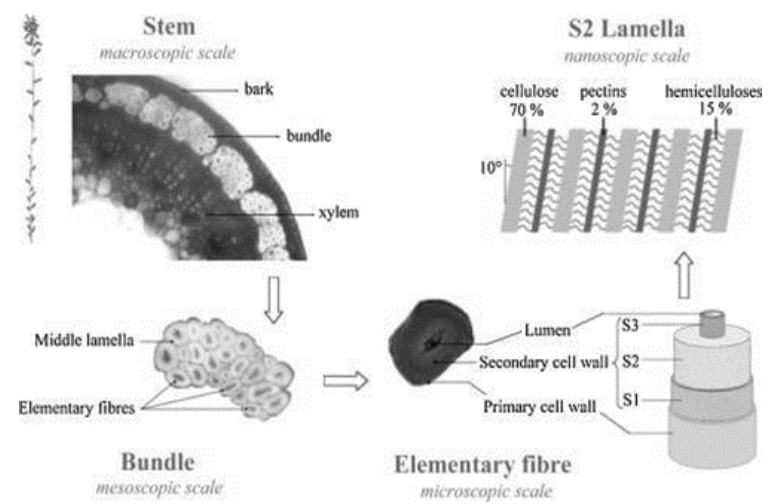

Figura 19. Physical Structure Recuperado de (Schlöesser, 2004)

La matriz junta las fibras, transfiere la carga, establece la forma de estructura y permite buenos acabados superficiales.

Esta estructura física puede ser modificada mediante el uso de procesos de tratamiento y de acetilación alcalinos. La aplicación de fibras naturales como refuerzos en materiales compuestos requiere, al igual que para materiales compuestos reforzados de fibra de vidrio, una fuerte adhesión entre la fibra y la matriz, independientemente de si un polímero tradicional (termoplástico o termoestable) de la matriz, una matriz de polímero biodegradable se utiliza.

\section{Modificación superficial}

Las fibras naturales incrementan su adhesión con la matriz del compuesto a través de tratamientos físicos y químicos, las modificaciones superficiales incluyen: tratamientos físicos, como extracción con disolventes; tratamientos físico-químicos, como el uso de descargas corona y plasma o láser, rayos- $\gamma$ y el bombardeo UV y las modificaciones químicas (Belgacem, 2005). 


\section{Propiedades mecánicas}

Al realizar la caracterización mecánica se opta por algunas pruebas, que se mencionan a continuación (Nur, 2010):

Prueba de Tensión: Este ensayo se utiliza para evaluar principalmente la resistencia última a la tensión, la elongación y módulo de Young de un material. La máquina generalmente usada es una Universal Tensile Testing Machine, con una capacidad aproximada de $10 \mathrm{kN}$ y velocidad de 1mm/min. El espécimen de la prueba fue preparado según ASTM D638-01 (2002) y la prueba estática de tensión fue realizada, siguiendo la misma norma.

Resistencia a la flexión: La norma generalmente usada es la ASTM D 790-00 (2002). Las propiedades de flexión de la fibra natural / PP materiales compuestos se caracterizaron usando ASTM D638-03.

Tenacidad: Prueba de impacto: Las normas más usadas para la realización de esta prueba son: ASTM D256-10 Determining the Izod Pendulun Impact resistance of plastics y ASTM D6110-10 Standard Test Method for Determining the Charpy Impact Resistance of Notched Specimens of Plastics.

Prueba de absorción de agua: se realiza conforme la norma ASTM D 570-99 (2002). Para esto las muestras se secan por aproximadamente 2 horas y a $105^{\circ} \mathrm{C}$. Se usa una balanza con precisión de $0.0001 \mathrm{~g}$. Se calcula el incremento en peso después de la inmersión en un recipiente de agua (boiling water) durante 2 horas.

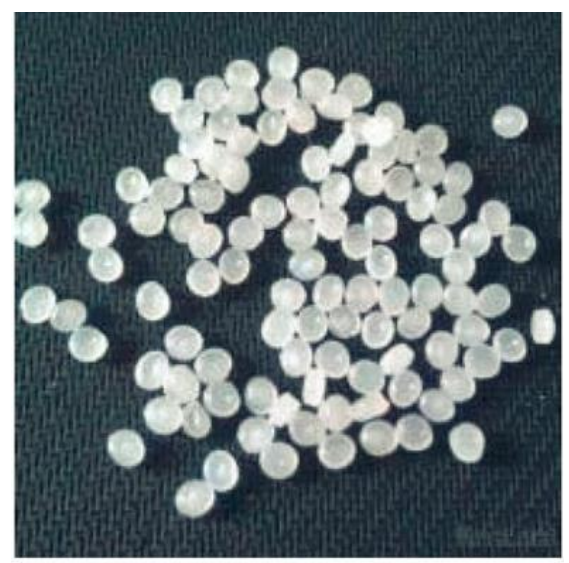

Figura 20. Image of polypropylene (PP) granules Recuperado de (Siddika, 2013) 


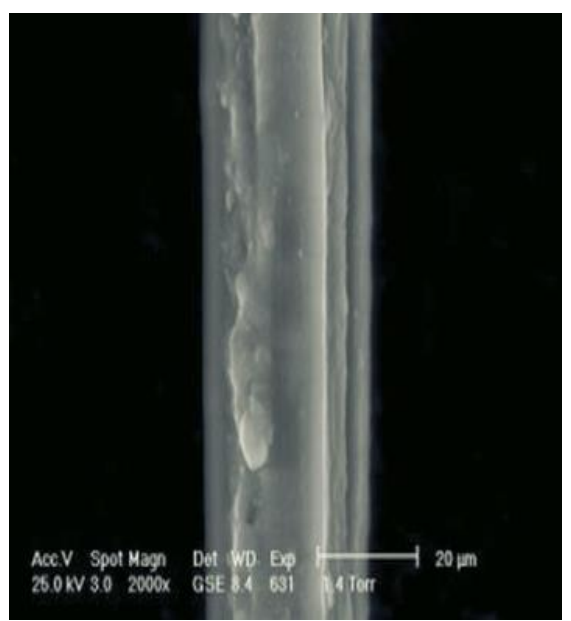

Figura 21. SEM image of juter fiber Recuperado de (Siddika, 2013)

En la Figura 20 puede observarse la forma en que se presenta el polipropileno y en la Figura 21 se observa la imagen de una fibra de yute (usando un microscopio)

\section{Microscopia Electrónica de Barrido: Scanning Electron Microscopy (SEM)}

Se basa en la toma de imágenes de alta resolución de diferentes muestras del material, utilizando un haz de electrones. Se pueden lograr acercamientos de hasta $1 \mathrm{~nm}$., usando instrumentos de alta resolución y de $3 \mathrm{~nm}$., usando instrumentos convencionales. Se usa para visualizar la superficie y analizar su estructura interna, al fracturar las muestras. Se pueden obtener imágenes desde diferentes ángulos y en 3D. Analizar sobre todo las superficies de fractura de los ensayos de tensión, principalmente. 


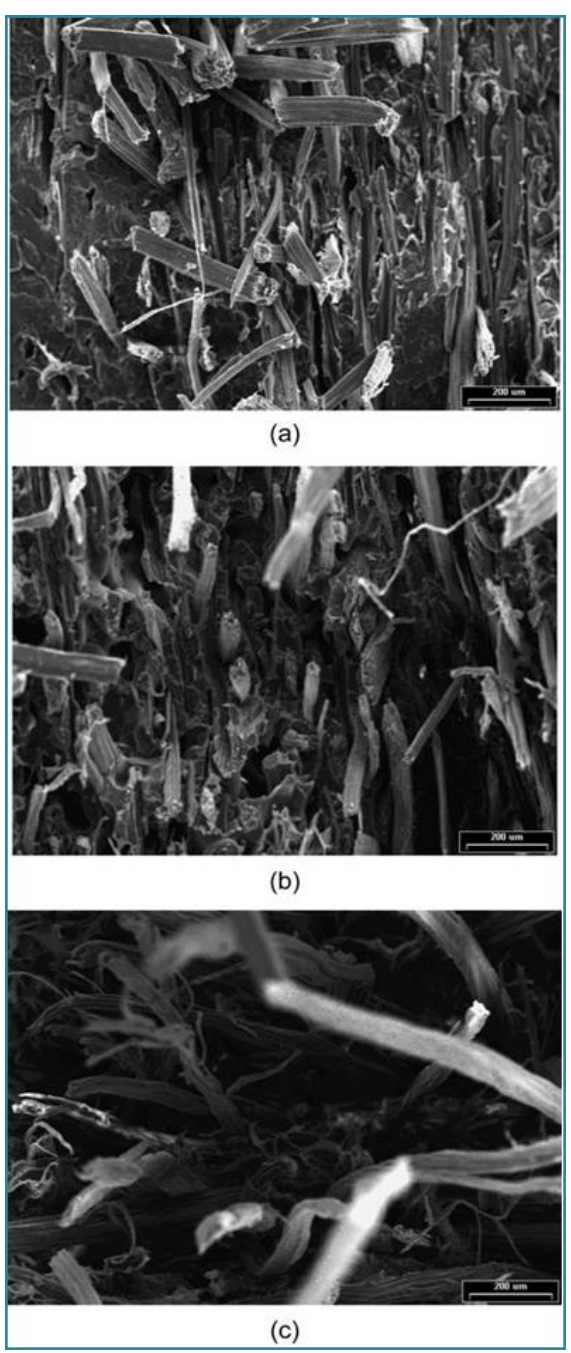

Figura 22. SEM micrographs of the fracture surface of jute fiber reinforced composite with nominal fiber fractions of (a) $30 \%$, (b) $50 \%$, and (c) $70 \%$. Recuperado de (Lee, 2009)

En la Figura 22 se puede observar la composición de la fibra y la matriz, luego de la fractura, analizado a diferentes porcentajes de proporción de la fibra.

La muestra tiene unas dimensiones de $2.54 \mathrm{~cm} \times 18.8 \mathrm{~cm} \times 0.8 \mathrm{~cm}$. Las fibras fueron cortadas de 3-5 mm de longitud. La humedad fue removida por un tiempo de 20 min a $80^{\circ} \mathrm{C}$.

\section{Resultados}

A continuación se analizan resultados obtenidos de diversas pruebas con algunos materiales.

\section{Propiedades de tensión:}

Analizando el Módulo de Young y Esfuerzo de Tensión, en cada una de las muestras del compuesto, con un contenido de 5, 10, 15 y 20\% para cada fibra, con la ayuda de la curva esfuerzo/deformación (Stress/Strain) para yute. 


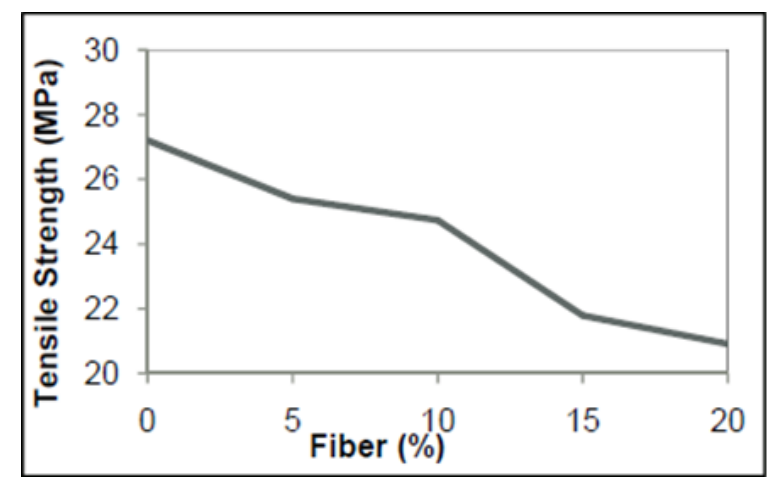

Figura 23. Variation of tensile strength at different fiber content Recuperado de (Siddika, 2013)

En la Figura 23 se puede apreciar que el valor de la resistencia a la tracción decrece con un incremento en la carga (porcentaje de fibra).

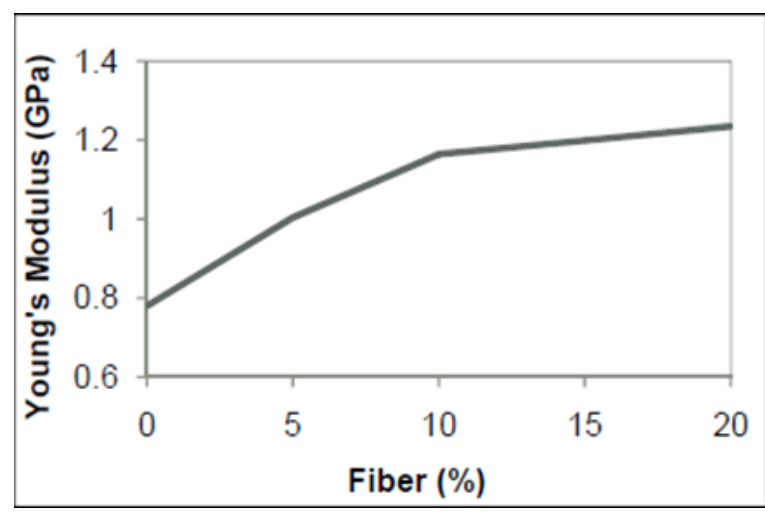

Figura 24. Variation of Young's modulus at different fiber content Recuperado de (Siddika, 2013)

La variación de la fuerza de tensión en función del porcentaje de fibra se observa en la Figura 24, lo cual nos muestra que a mayor porcentaje de fibra de este tipo menor valor de la fuerza de tensión.

En lo referente a la resistencia a la flexión, podemos observar en la Figura 25, como el porcentaje mayor de fibra en la matriz ayuda a elevar esta propiedad.

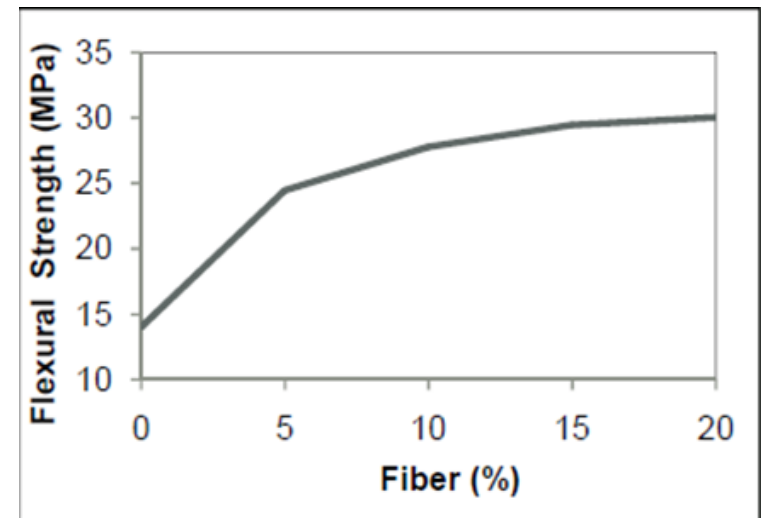

Figura 25. Variation of flexure strength at different fiber content Recuperado de (Siddika, 2013) 


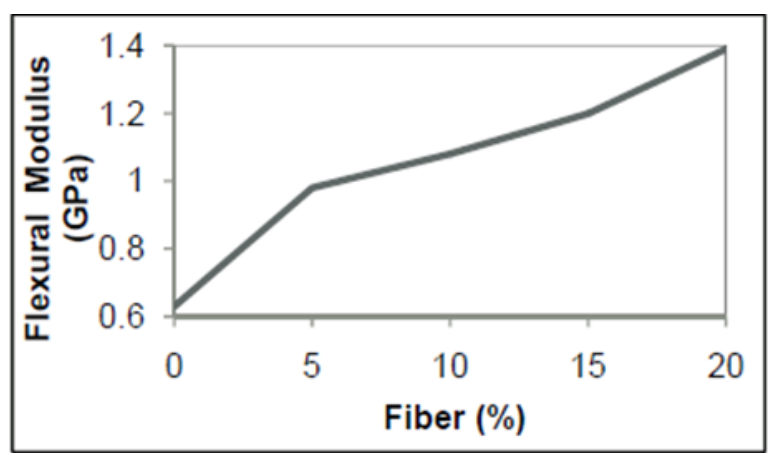

Figura 26. Variation of flexure modulus at different fiber content Recuperado de (Siddika, 2013)

La Figura 26 muestra que así mismo el Modulo de Flexión aumenta con el porcentaje de carga (fibra).

\section{Fuerza de impacto: Impact Strength}

Una variación de la prueba de Charpy, en función del porcentaje de fibra se observa en la Figura 27.

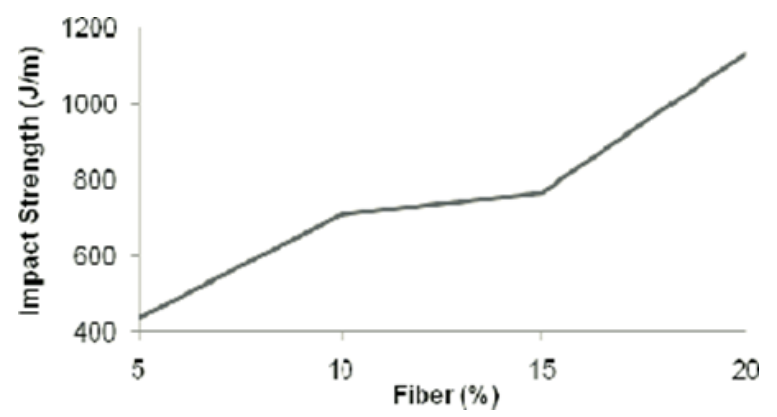

Figura 27. Variation impact strength at different fiber content Recuperado de (Siddika, 2013)

Dureza Depende de la distribución de la carga en la matriz y una mejor dispersión de la carga en la matriz con minimización de huecos.

La Figura 28 muestra los valores de las fuerzas de tensión, compresión y flexión, para algunos compuestos, donde se puede apreciar que al usar cualquier tipo de fibra natural, las propiedades mecánicas del material no varían mucho, y están acordes a las necesidades exigidas en la fabricación de la pieza. 


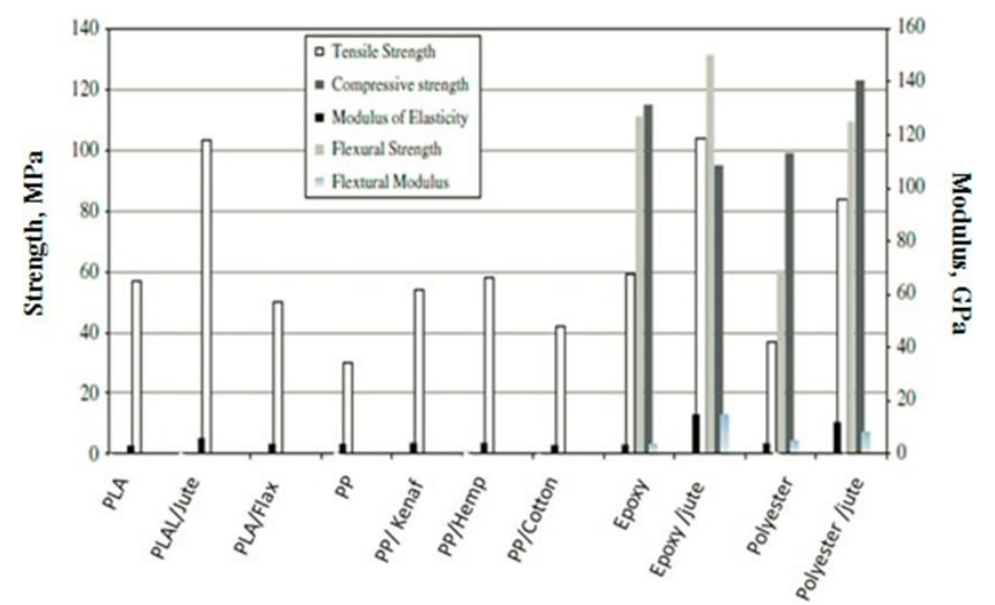

Figura 28. Some of mechanical properties of natural fiber reinforced polymer composite Recuperado de (Westman, 2010)

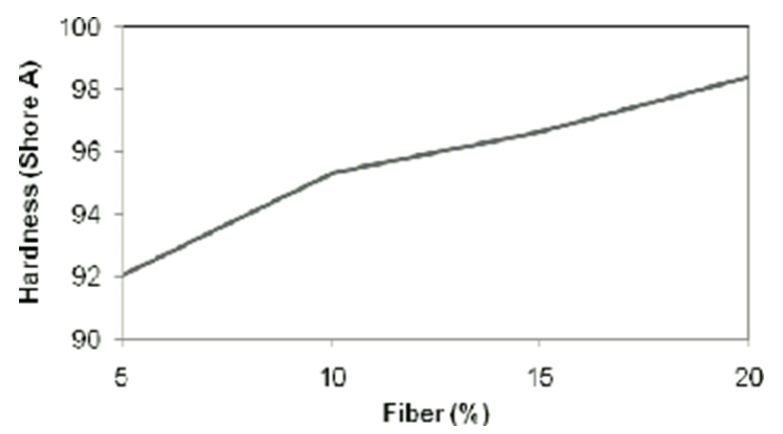

Figura 29. Variation of hardness at different fiber content Recuperado de (Siddika, 2013)

La fuerza al impacto (Figura 27) y la dureza (Figura 29), también aumentan en función de la cantidad (\%) de fibra existente en el compuesto.

\section{Morfología de la superficie}

La Figura 30 nos muestra la fibra con tratamiento y sin tratamiento.

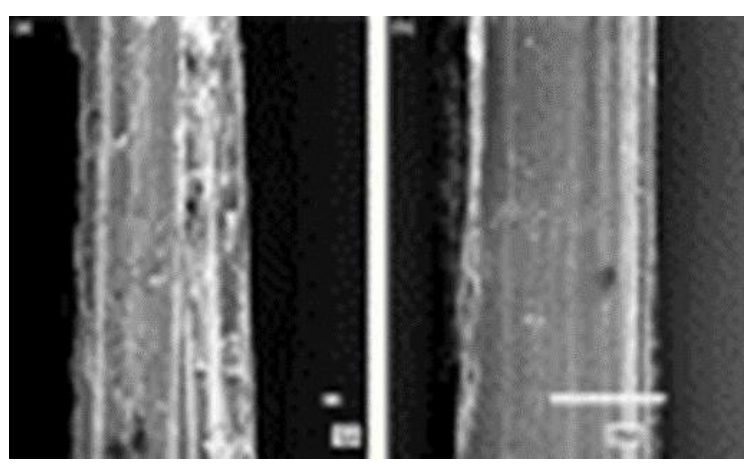

Figura 30. Fibers Recuperado de (Nur, 2010)

En la Figura 31 observamos el material para el procesamiento al inicio, terminada su primera fase y el resultado final del panel interior para una puerta del automóvil. Las 
especificaciones del material son $1600 \mathrm{~g} / \mathrm{m} 2,50 \%$ de fibra- $50 \%$ de polipropileno (Holbery, 2006).

Las superficies de fractura del yute / PP compuestos eran investigado utilizando la micrografía SEM en la Figura 32. La yute / PP material compuesto con una fracción de fibra nominal de $30 \%$ (en peso) contiene matriz de PP más continua que cualquiera de los más altos de la fracción de fibra-compuestos. Las fibras de yute en Figura 32 (a) parece estar rodeado por la matriz de PP, mientras que algunos de ellos se retiraron durante el ensayo de tracción. Nota que los huecos se pueden observar en la Figura 32 (b) y (c).

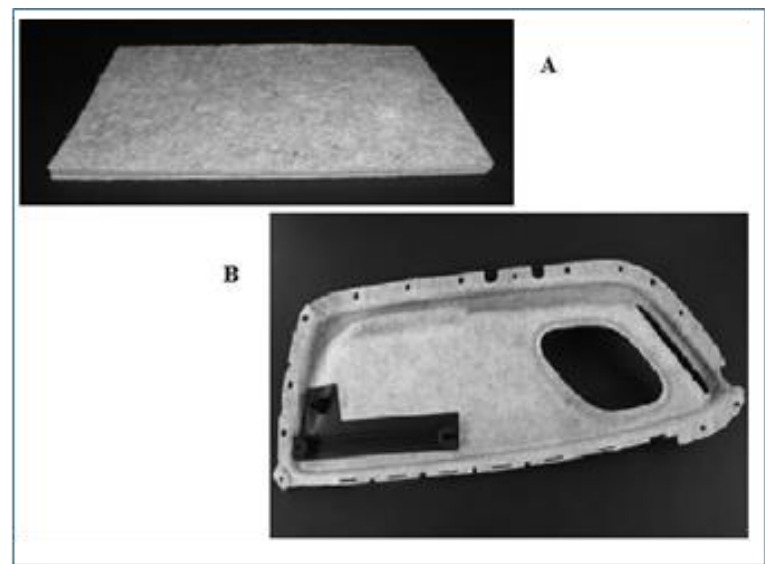

Figura 31. Inner Panel Doors Adaptado de (Ayrilmis, 2011)

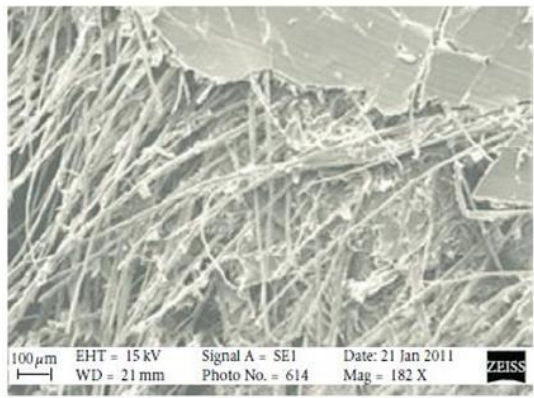

(a)

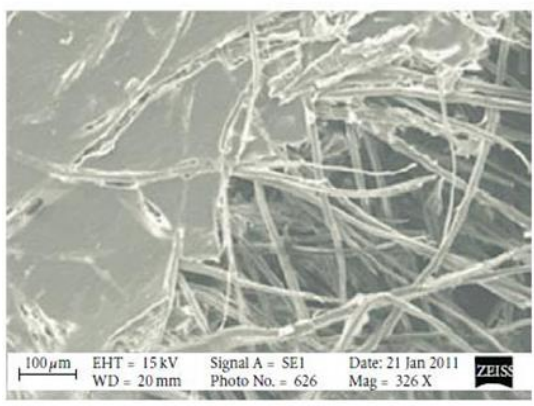

(c)

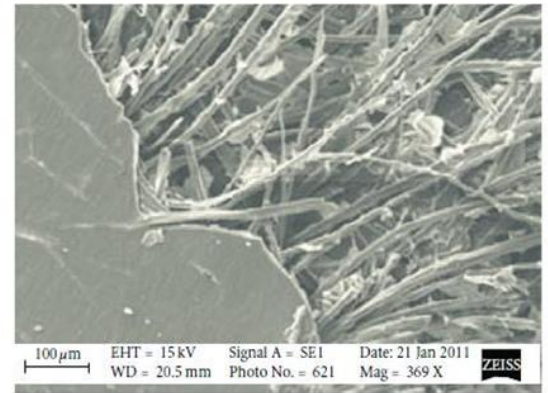

(b)

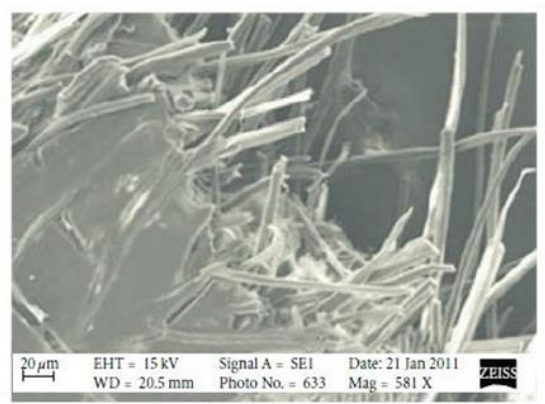

(d)

Figura 32. SEM images of the fracture surface of the PP composites reinforced with kenaf fibers digested at (a) $80^{\circ} \mathrm{C}$, (b) $110^{\circ} \mathrm{C}$, (c) $130^{\circ} \mathrm{C}$, and (d) $160^{\circ} \mathrm{C}$. Adaptado de (Aziz, 2004) 


\section{Conclusiones}

El material para aplicaciones de uso automotriz, en interiores puede ser uno del tipo matriz de material polimérico (Termofijo o Termoplástico) con reforzamiento de fibras sintéticas o naturales.

Para nuestro caso el material seleccionado para la fabricación de paneles internos de las puertas de un automóvil es un material polimérico (polipropileno) con un reforzamiento de fibra natural que es yute.

El compuesto brinda muchas ventajas, siendo las principales buenas características mecánicas, propiedades físicas acordes a la aplicación, bajo costo de fabricación, buen acabado.

Las condiciones de servicio definidas para los paneles internos, tienen relación directa con las dimensiones del habitáculo y condiciones de seguridad, en impactos de tipo lateral.

La implementación de este tipo de materiales en la fabricación de piezas automotrices, es muy frecuente, por lo relacionado a las normas de reciclado y reusado de las partes de los automóviles.

Las fibras naturales pueden sustituir a las fibras sintéticas (Sustitución de fibra de vidrio), especialmente para la fabricación de partes del interior del coche como: paneles de puertas, estantes de paquetería, y apoyacabezas.

Se ha observado que, además de su alta resistencia y rigidez por unidad de peso y las virtudes ambientales, los materiales tienen también otras ventajas. Estos incluyen el aislamiento acústico, fácil gestión de la salud y la seguridad, la producción rápida por compresión o moldeo por inyección, y potencialmente menor costo.

A mayor porcentaje de fibra natural en el compuesto mejor las prestaciones mecánicas, en nuestro caso definimos un máximo de $20 \%$ por las solicitaciones de los componentes internos, que son menores alas exteriores.

El porcentaje en disminución del peso de los componentes al usar las fibras naturales con polímeros, oscila alrededor de un 20-30\%.

Para mejorar la unión (cohesión) entre la fibra y la matriz (PP) se pueden usar algunos métodos de tratamiento.

El principal inconveniente que tienen estos materiales es la temperatura que puede alcanzar la fibra $180-200^{\circ} \mathrm{C}$ y otro la cantidad de humedad que tiene la fibra.

Para solventar problemas de humedad de la fibra se puede realizar tratamientos de secado y optimización del proceso. 
Los factores importantes considerados en la elección del material, para esta aplicación específica, es el bajo costo, factor ecológico y disminución de peso.

Se consideró los esfuerzos a tensión e impacto, mismos que deben estar relativamente bajos, si los comparamos con elementos externos del vehículo.

Los componentes químicos de la fibra son otro factor importante (cantidad de celulosa) tipo de fibra, que en muchos casos dependen de factores como clima y tipo de cultivo.

En el desarrollo de este tipo de materiales, se está poniendo mucho énfasis y presupuesto, por parte de los investigadores y fabricantes de vehículos.

Hay que dirigir la fabricación de compuestos con fibras naturales a obtener propiedades superiores, que incluye la modificación química eficaz, eficiente y económica de la fibra, la modificación de la matriz de funcionalización y la combinación de técnicas de procesamiento.

\section{Bibliografía}

Ahmad, F. C. (2015). A review: natural fiber composites selection in view of mechanical, light weight, and economic properties. . Macromolecular Materials and Engineering, 300(1), 1024.

Ashby, M. F. (1989). Materials selection in conceptual design. Materials science and technology.

Ayrilmis, N. J. (2011). Coir fiber reinforced polypropylene composite panel for automotive interior applications . Fibers and polymers, 12(7), 919-926.

Aziz, S. H. (2004). The effect of alkalization and fibre alignment on the mechanical and thermal properties of kenaf and hemp bast fibre composites: part 2-cashew nut shell liquid matrix. Composites Science and Technology, 64(9), 1231-123.

Belgacem, M. a. (2005). The surface modification of cellulose fibres for use as reinforcing elements in composite materials. Composite Interfaces, , 12(1-2): p. 41-75.

Cristaldi, G. L. (2010). Composites based on natural fibre fabrics. . Woven fabric engineering, 317-342.

ElayaPerumal, A. \&. (2008). Natural Fiber-Reinforced Polymer Composites in Automotive Applications-A Review. . IJAEA, 1(6), 68-74.

Faruk, O. B. (2012). Biocomposites reinforced with natural fibers . Progress in Polymer Science, 37(11), 1552-1596.

Faruk, O. B. (2014). Progress report on natural fiber reinforced composites . Macromolecular Materials and Engineering, 299(1), 9-26. 
Faruk, O. B. (n.d.). Biocomposites reinforced with natural fibers: 2000-2010. Progress in Polymer Science, 2012. 37(11). p1552-1596.

George, J. S. (2001). A review on interface modification and characterization of natural fiber reinforced plastic composites. Polymer Engineering \& Science, 41(9), 1471-1485.

Hill, K. S. (2012). The bio-based materials automotive value chain. Center for Automotive Research, 112.

Holbery, J. \&. (2006). Natural-fiber-reinforced polymer composites in automotive applications. Jom, 58(11), 80-86.

http://www.crc-acs.com.au/index.php/news/12-main-articles/technical-articles/148-technicalarticlenatural-fibre-composites. (n.d.).

http://www.globalhemp.com/2011/02/automotive-composites.html. (n.d.).

http://www.nhtsa.gov/cars/rules/import/FMVSS/. (n.d.).

https://www.euroncap.com/es. (n.d.).

John, M. J. (2008). Recent developments in chemical modification and characterization of natural fiber-reinforced composites. . Polymer composites, 29(2), 187.

Kinoshita, A. S. (2012). Development of Pole Side Impact Sled Test Method using Multiple Actuators for EuroNCAP, . SAE Technical Paper 2012-01-0095 , doi:10.4271/2012-010095 .

Lee, B. H. (2009). Fabrication of long and discontinuous natural fiber reinforced polypropylene biocomposites and their mechanical properties. Fibers and Polymers, 10(1), 83-90.

Majewski, T. \&. (n.d.). Desarrollo y aplicaciones actuales de los plásticos reforzados por fibras naturales. reproducción, $2,4$.

Mohanty, A. K. (2002). Sustainable bio-composites from renewable resources: opportunities and challenges in the green materials world. Journal of Polymers and the Environment, 10(1-2), 19-26.

Monteiro, S. N. (2009). Natural-fiber polymer-matrix composites: cheaper, tougher, and environmentally friendly. Jom, 61(1), 17-22.

Mueller, D. H. (2002, September). Acoustical properties of reinforced composite materials and layered structures basing on natural fibers. . In Proceedings of the INTC-International Nonwov. 
Mueller, D. H. (2004). Improving the impact strength of natural fiber reinforced composites by specifically designed material and process parameters . Int. Nonwovens J, 13(4), 31-38.

Nur, H. P. (2010). Nur, H. P., Hossain, M. A., Sultana, S., \& Mollah, M. M. (2010). Preparation of polymer composites using natural fiber and their physico-mechanical properties. Bangladesh Journal of Scientific and Industrial Research, 45(2), 117-122.

Pan, N. (1993). Theoretical determination of the optimal fiber volume fraction and fiber-matrix property compatibility of short fiber composites. Polymer composites. 14(2), 85-93.

Saheb, D. N. (1999). Natural fiber polymer composites: a review. . Advances in polymer technology, 18(4), 351-363.

Satyanarayana, K. G. (2007). Studies on lignocellulosic fibers of Brazil. Part I: Source, production, morphology, properties and applications. Composites Part A: Applied Science and Manufacturing, . 38(7).

Schlöesser, T. P. (2004). Natural fiber reinforced automotive parts. In Natural Fibers, Plastics and Composites. Springer US., (pp. 275-285).

Siddika, S. M. (2013). Physico-Mechanical Properties of Jute-Coir Fiber Reinforced Hybrid Polypropylene Composites. World Academy of Science, Engineering and Technology, 73, 11451149.

Suddell, B. C. (2008, October). Industrial fibres: recent and current developments. In Proceedings of the Symposium on Natural Fibres. . Organized by FAO and CFC, (pp. (Vol. 20, pp. 71-82).). Rome.

Taj, S. M. (2007). Natural fiber-reinforced polymer composites. Proceedings-Pakistan Academy of Sciences, 44(2), 129.

Westman, M. P. (2010). Westman, M. P., Fifield, L. S., Simmons, K. Natural Fiber Composites: A Review (No. PNNL-19220). . Pacific Northwest National Laboratory (PNNL), Richland, WA (US). 